\title{
A real-time ultrasonic field mapping system using a Fabry Pérot single pixel camera for 3D photoacoustic imaging
}

\author{
Nam Huynh ${ }^{1}$, Edward Zhang ${ }^{1}$, Marta Betcke ${ }^{2}$, Simon Arridge ${ }^{2}$, Paul Beard ${ }^{1}$ and Ben Cox ${ }^{1}$ \\ ${ }^{1}$ Department of Medical Physics and Bioengineering, University College London, WC1E 6BT. UK \\ ${ }^{2}$ Department of Computer Science, University College London, WC1E 6BT. UK
}

\begin{abstract}
A system for dynamic mapping of broadband ultrasound fields has been designed, with high frame rate photoacoustic imaging in mind. A Fabry-Pérot interferometric ultrasound sensor was interrogated using a coherent light single-pixel camera. Scrambled Hadamard measurement patterns were used to sample the acoustic field at the sensor, and either a fast Hadamard transform or a compressed sensing reconstruction algorithm were used to recover the acoustic pressure data. Frame rates of $80 \mathrm{~Hz}$ were achieved for $32 \times 32$ images even though no specialist hardware was used for the on-thefly reconstructions. The ability of the system to obtain photocacoustic images with data compressions as low as $10 \%$ was also demonstrated.
\end{abstract}

Keywords: Photoacoustic imaging, digital micromirror array, ultrasound field mapping, real time, compressed sensing

\section{INTRODUCTION}

Biomedical photoacoustic imaging ${ }^{1}$, relies on the generation of broadband ultrasonic waves following the absorption and thermalisation of a short laser pulse within a region of optical absorption in tissue. Measuring photoacoustic waves, or indeed any ultrasound field, accurately requires arrays (or synthesised arrays) whose element sizes and spacings are, ideally, smaller than the shortest wavelength measurable and with sufficient sensitivity over the bandwidth of interest. For broadband detection over tens of $\mathrm{MHz}$ frequencies, such as are typically generated in biomedical photoacoustics, there are only a few candidates that satisfy these requirements. Non-optical approaches for detecting high frequency ultrasound include capacitive ${ }^{2}$ and piezoelectric devices, ${ }^{3,4}$, although they are typically limited in bandwidth and sensitivity at small element sizes, and the fabrication costs of arrays of more than a few hundred elements soon becomes prohibitive. Optical approaches, which typically exploit optical resonance within the sensing device to obtain high sensitivity, have shown greater promise. Examples include microring resonators ${ }^{5}$, domed microresonators ${ }^{6}$ and FabryPérot (FP) etalons ${ }^{7-9}$. The latter have been shown to exhibit very wide bandwidths $(>100 \mathrm{MHz})$, and exhibit high sensitivity at small element sizes $(<1 \mathrm{~mm})$.

Fabry-Pérot ultrasound sensors interrogated using point-by-point scanning ${ }^{7,10}$ have been used for many years in photoacoustic imaging, and have been shown to give exquisitely detailed images ${ }^{11}$. However, the speed of the data acquisition is limited by the requirement to scan which limits it to imaging static or very slowly moving photoacoustic images. To overcome this, multi-pixel cameras ${ }^{12-14}$ have been proposed, but they have low sampling rates or can measure at only a few time points. It is demonstrated in this paper that by interrogating it using patterns set using a digital micromirror device (DMD) ${ }^{15}$, and working within a compressed sensing framework, allows real time capture of dynamically changing ultrasound fields. This is a significant step towards high resolution, three dimensional, dynamic photoacoustic imaging.

Photons Plus Ultrasound: Imaging and Sensing 2015, edited by Alexander A. Oraevsky, Lihong V. Wang

Proc. of SPIE Vol. 9323, 932310 - C) 2015 SPIE · CCC code: 1605-7422/15/\$18

doi: $10.1117 / 12.2081869$

Proc. of SPIE Vol. 9323 932310-1 


\section{2.}

\section{R DESIGN AND OPERATION}

To grasp the basic idea behind interrogating the FP sensor using a DMD rather than a single focussed beam, consider the relationship between the time-varying acoustic field at a set of $N$ points on the FP sensor plane, $p_{1}(t), \ldots, p_{N}(t)$, and the time series that are measured, $s_{1}(t), \ldots, s_{N}(t)$ :

$$
\left[\begin{array}{c}
s_{1}(t) \\
\vdots \\
s_{N}(t)
\end{array}\right]=\left[\begin{array}{ccc}
m_{11} & \ldots & m_{1 N} \\
\vdots & \ddots & \vdots \\
m_{31} & \ldots & m_{N N}
\end{array}\right]\left[\begin{array}{c}
p_{1}(t) \\
\vdots \\
p_{N}(t)
\end{array}\right], \quad \mathbf{s}(t)=\mathbf{M p}(t) .
$$

For the case of the point scanner, the matrix $\mathbf{M}$ is to a good approximation the identity matrix; the signals measured correspond to the time series at chosen points on the sensor. When interrogating with the DMD scanner, however, the matrix $\mathbf{M}$ is a scrambled Hadamard matrix, where each row is a (reordered version of a) binary scrambled Hadamard pattern; see Fig. 1. Each time series measurement with the DMD is therefore the summation of the time varying pressure at many points on the sensor. The advantage of this method of sampling the field is that it is amenable to the techniques of compressed sensing (see Section 2.2 below).

\subsection{Experimental Set-up}

The Fabry-Perot ultrasound sensor (aluminium mirror coatings, $50 \mu \mathrm{m}$ parylene spacer, glass substrate) was interrogated in a region of uniform sensitivity by a 2 $\mathrm{cm}$ diameter beam (Santec TSL-510 tunable laser source, IPG Laser GmbH

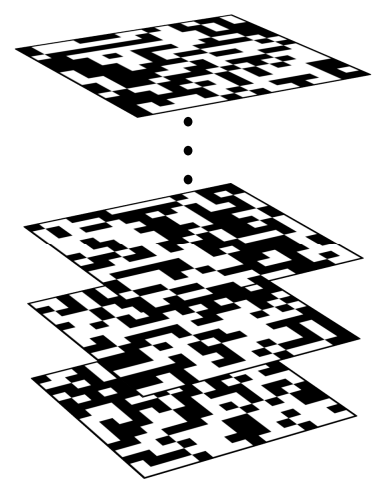

Figure 1: Scrambled Hadamard matrices were used as measurement patterns.

Erbium Fiber Amplifier EAD-4-L). The acoustic field at the sensor was thereby encoded onto the intensity of the reflected light. The light was then reflected from the DMD (ViaLUX V-7000vis DLP, TI 0.7" XGA, 1024x768 micromirror array, pitch $13.68 \mu \mathrm{m}$, maximum binary frame rate $22 \mathrm{kHz}$ ) which patterned it sequentially with the chosen measurement patterns, here scrambled Hadamard matrices. The strongest diffracted order from the $\mathrm{DMD}^{16}$ was collected by a lens (focal length $25.4 \mathrm{~mm}$ ) into a customised InGaAs photodiode (G8376-03), which is equivalent to performing an analogue spatial integral over the illuminated area of the FP sensor. This arrangement is shown schematically in Fig 2. An oscilloscope (NI PCI-5114 digitiser, 50MHz sampling rate and 20MHz bandwidth) was used to acquire the data, and LABVIEW was used to control the whole system.

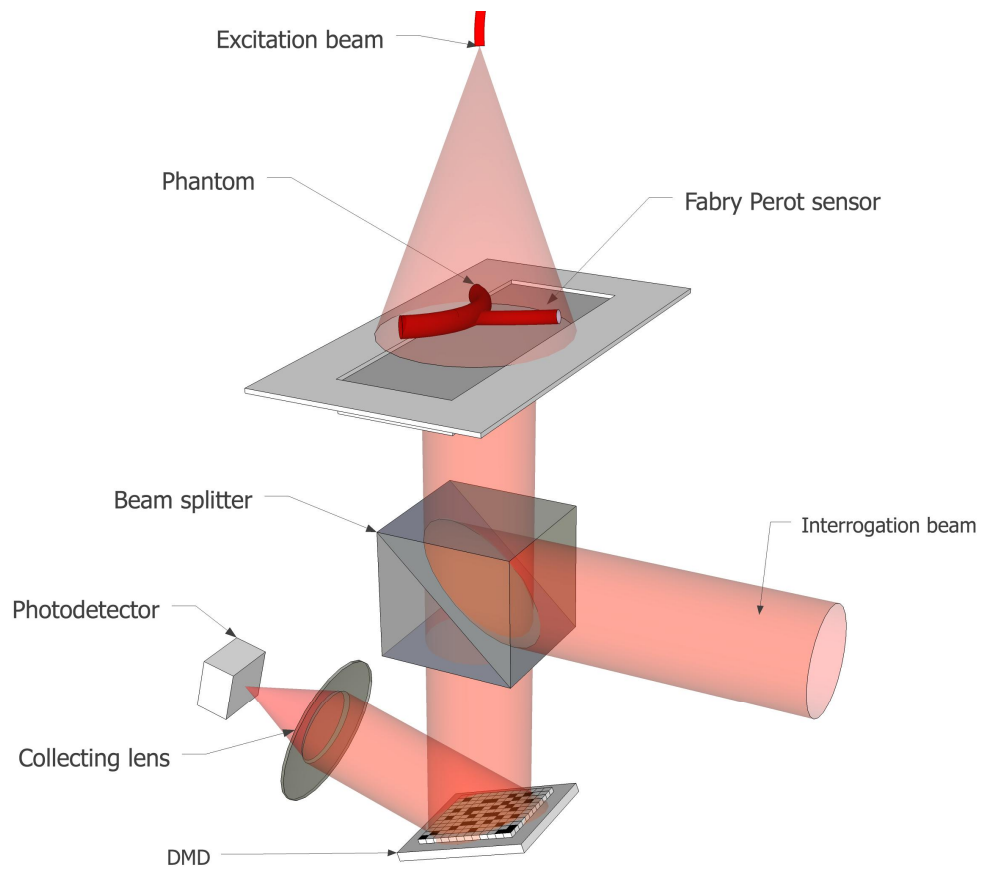

Figure 2: Experimental arrangement for a photoacoustic imaging experiment using the Fabry-Perot single-pixel camera system. This arrangement, but with the photoacoustic source replaced by a focussed ultrasound transducer, was used for the dynamic field mapping experiments. 


\subsection{Compressed sensing using scrambled Hadamard matrices}

Consider a 2D function $p(x, y)$, which represents the acoustic pressure on the surface of the FP sensor at a certain time. The Nyquist-Shannon sampling theorem says that for a bandlimited function, ie. one with a finite maximum wavenumber $k_{\max }$, the exact function can be reconstructed from samples of the function if and only if the spatial sampling frequency, $k_{s}$, is greater than twice the highest wavenumber component, $k_{s}>2 k_{\max }$. By using compressed sensing, this limit can beaten when the function is known to have some structure, ie. it is sparse in some basis (eg. if it is non-zero at only a few points, or consists of just a few wavenumber components). In other words it is possible to recover it exactly using fewer measurements than Nyquist requires; however, the sampling scheme must satisfy certain conditions (such as incoherence to the sparse basis) ${ }^{17}$. For spatial sampling in 2D it is known that scrambled Hadamard matrices - Hadamard matrices whose rows and columns have been permuted randomly - have nearly optimal performance, in the sense that the number of measurements required for a perfect reconstruction is close to the theoretical bound, and they can be used with many different sparse bases ${ }^{18,19}$ (see Fig. 1). When using compressed sensing, the first $M<N$ rows of the $N \times N$ Hadamard matrix were used (so $M$ randomly selected patterns).

Two measurement schemes were used in the dynamic field mapping experiments. In the first mode the data was measured, reconstructed using the fast Hadamard transform (with zeros replacing missing data as necessary), and displayed for each frame before the data for the next frame was measured. In the second mode, the data was measured continuously, with the patterns cycling through as necessary, without any breaks for reconstruction. The acoustic pressure values on the sensor at time $t, p_{t}=\Psi a_{t}$, were then found by optimising the fit to the data whilst subject to a constraint enforcing sparsity in the basis $\Psi$. The SALSA algorithm ${ }^{20}$ was used to solve the minimisation:

$$
a_{t}=\underset{a_{t}}{\arg \min } \frac{1}{2}\left\|\Phi \Psi a_{t}-w_{t}\right\|_{2}^{2}+\alpha\left\|a_{t}\right\|_{1}
$$

where $\Phi$ are the scrambled Hadamard measurement patterns, $w_{t}$ the measured data, and $\alpha$ is a regularisation parameter. Suitable choices for the sparsity basis $\Psi$ include curvelets, wavelets and the identity matrix used here.

\section{DYNAMIC FIELD MAPPING}

To demonstrate the ability of the system to map dynamically moving acoustic fields in real time, a focussed ultrasound transducer (Panametrics V319, $15 \mathrm{MHz}, 0.5$ " diameter, focal length 0.75 ") was positioned above the FP sensor with the focus close to the surface. It was driven by a power amplifier (Precision Acoustics, PA25-150) with the signal provided by a signal generator (Agilent 33210A). The transducer focus was then moved manually to and fro across the sensor while the system was mapping the ultrasonic field. To demonstrate the three-dimensional $(x, y, t)$ nature of the data that is recorded for each frame, and, more specifically, how changes in all 3 dimensions can be captured, the duration of the tone burst - the number of cycles in the pulse - was changed in some of the examples.

\subsection{Example 1: Offline reconstruction, $148 \mathrm{~Hz}$ frame rate}

In this example the ultrasound transducer was driven with a 5 cycle tone burst. The system recorded and stored the data for offline processing. For a $32 \times 32$ pixel image, 128 patterns, or time series, were recorded ( $12.5 \%$ compression). While the ultrasound focus was moved across the sensor surface 400 consecutive data frames of 128 time series each were recorded. The full set of time series data is shown in Fig. 3. There is no variation in the time of arrival of the pulse as the transducer remained parallel to the sensor plane while it was moved laterally. The variation in position between frames is not discernible from the time series, but can be seen in the reconstructed frames. The time series for each frame were reconstructed into a $3 \mathrm{D}$ data set of acoustic pressure on the sensor, $p(x, y, t)$; maximum intensity projections of $p$ over time are also shown in Fig. 3 for frames 50, 290 and 388. The maximum DMD pattern refresh rate is $22 \mathrm{kHz}$ ( $20 \mathrm{kHz}$ was used here); the frame rate is limited by the number of patterns that are required per frame, and that is dictated by the number of pixels required per frame and the compression that is achievable. The frame rate achieved here was $148 \mathrm{~Hz}$. 


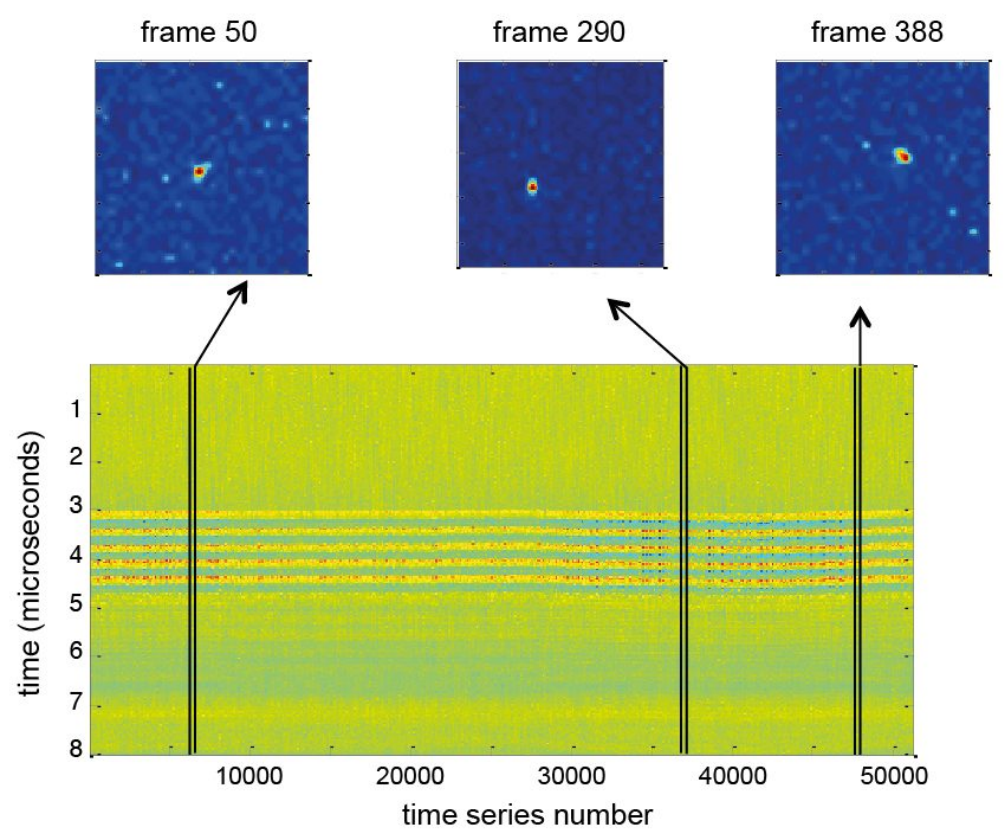

Figure 3: Example 1, dynamic field mapping of the moving focus from an ultrasound transducer. The full set of measured time series and 3 of the 400 consecutively recorded frames are shown.

\subsection{Example 2: Offline reconstruction, $80 \mathrm{~Hz}$ frame rate (movie)}

This example is similar to Example 1 except that the toneburst duration was varied every $20 \mathrm{~ms}$ (a rate of $50 \mathrm{~Hz}$ ) so that the advantage of using compressed sensing could be seen. In movie example_2a each $32 \times 32$ pixel frame was recovered from 1024 measurements (no compression) giving a frame rate of only $20 \mathrm{~Hz}$, whereas in movie example_2b just 256 measurements $(25 \%$ compression) were used giving a frame rate of $80 \mathrm{~Hz}$. The former is not fast enough to capture the $50 \mathrm{~Hz}$ variation in the toneburst duration but in the latter case it is, clearly showing the advantage of using compressed sensing to achieve a higher frame rate. (The reconstructions in this example were with the fast Hadamard transform.)

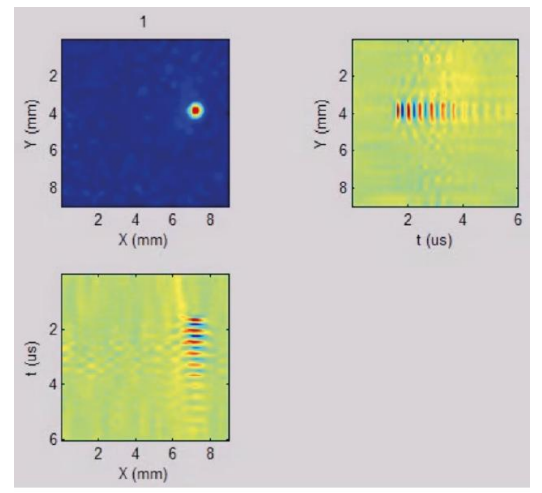

Movie 1: example 2a http://dx.doi.org/10.1117/12.2081869.1

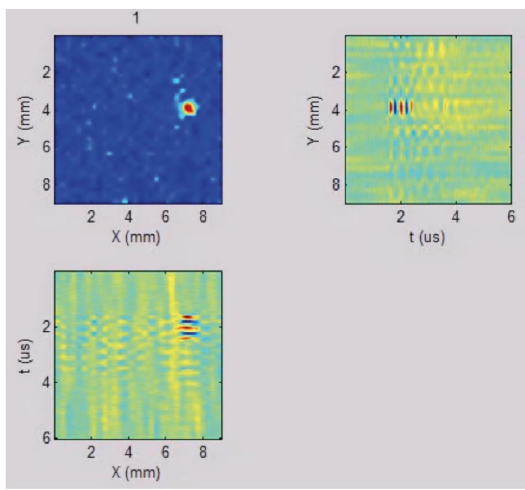

Movie 1: example_2b http://dx.doi.org/10.1117/12.2081869.2 


\subsection{Example 3: Reconstruction on-the-fly, $9 \mathrm{~Hz}$ frame rate (movie)}

This example is similar to Example 2 except that instead of reconstructing the data offline it was done on-the-fly between frames. Also the toneburst duration was changed every $100 \mathrm{~ms}(10 \mathrm{~Hz})$. Using dedicated hardware it would, in principle, be possible to achieve real time frame rates limited only by the DMD refresh rate. In practice, without such hardware, the real time frame rates achievable are limited by the time taken for the signal reconstruction (the fast Hadamard transform) which here was implemented in Labview calling Matlab functions. In this experiment, the system recorded, processed and displayed the acoustic field in real time at a frame rate of $9 \mathrm{~Hz}$. The movie example_3 shows that the variation in the position and the toneburst duration have been captured.

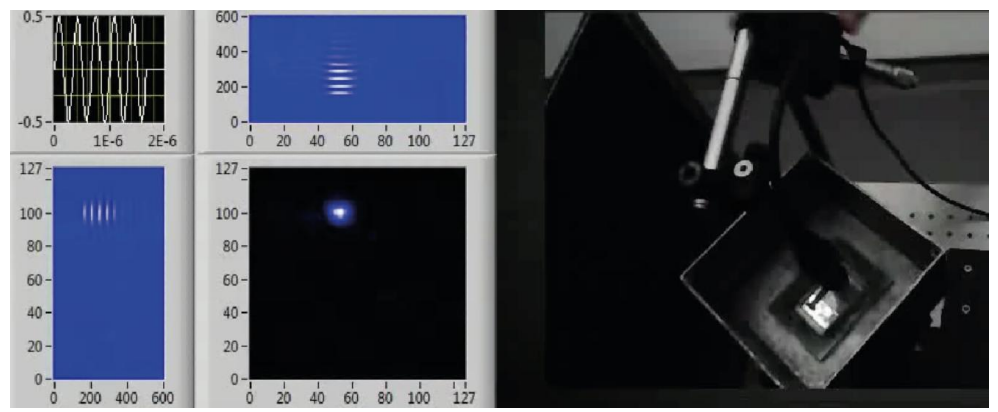

Movie 3: example_3, http://dx.doi.org/10.1117/12.2081869.3

\subsection{Example 4: Reducing the motion artifacts with compressed sensing}

This final example demonstrates that compressed sensing can reduce blurring artifacts due to motion of the source, by allowing a faster frame rate which can capture the faster movement. A focused tone burst is moved across the sensor, and 1024 measurements were made (for an image with 32x32 pixels). In movie example $4 a$ the frames were reconstructed using 1024 measurements, but in order to increase the frame rate frames were calculated with sets of 1024 measurements overlapping by 256 frames. In other words, the data used in a frame has only 256 new measurements and the other 768 measurements were shared with the previous measurement; a measurement can contribute to 4 sequential images. Motion artifacts - a lag or tail to the moving spot - is clearly visible in the movie. In contrast, example_ $4 b$ was reconstructed using non-overlapping sets of just 256 measurements. In this case the lag has gone and the focussed spot is not blurred across frames at different times. In these movies the reconstruction was done using the fast Hadamard transform. A similar result (the same arrangement but a different data set and reconstructed using the SALSA algorithm) is shown in the maximum intensity projections in Fig. 4. The images on the left (constructed with overlapping data) suffer from considerable blurring due to the movement of the focus. In the images on the right this has been avoided by using non-overlapping data and a compressed sensing reconstruction.

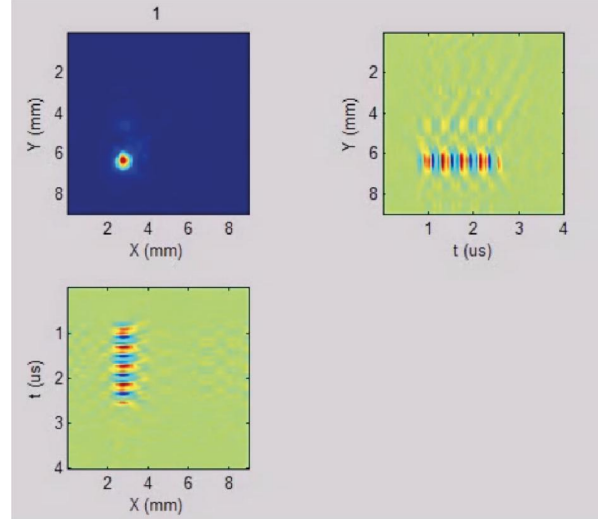

Movie 4: example_4a http://dx.doi.org/10.1117/12.2081869.4

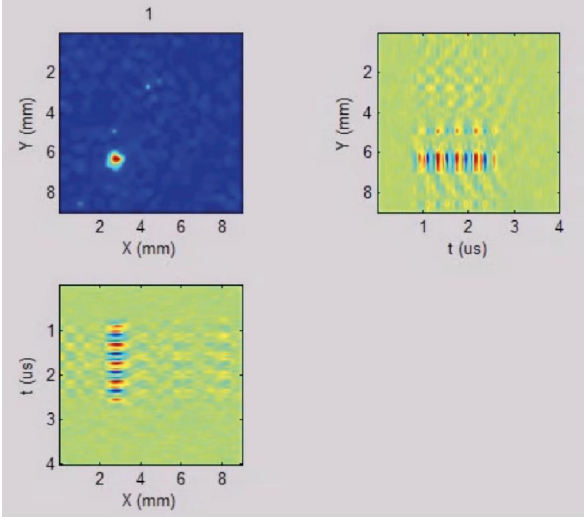

Movie 5 example_4b http:// dx.doi.org/10.1117/12.2081869.5 

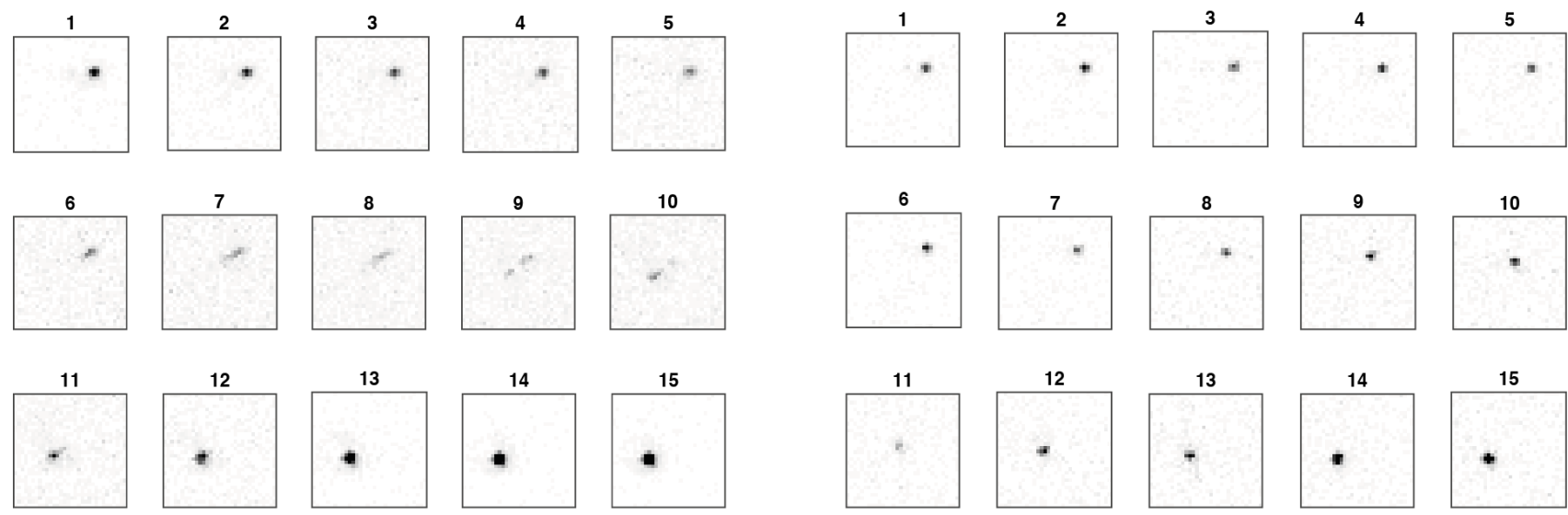

Figure 4: Ultrasound focus moving from top right towards bottom left. (Left) Frames reconstructed using sets of 1024 measurements overlapping by 768 measurements showing considerable motion blur in frames 6-11. (Right) Frames reconstructed using compressed sensing using non-overlapping sets of 256 measurements exhibiting no motion blur.

\section{PHOTOACOUSTIC IMAGING}

One application for dynamic ultrasound field mapping is photoacoustic imaging ${ }^{1}$, in which ultrasound fields are generated through the absorption of short pulses of light. By recording these acoustic emissions on the tissue surface it is possible to infer the initial distribution of absorbed optical energy and hence the presence of absorbing structures, such as blood vessels. To make full use of a dynamic mapping system such as this for photoacoustics will require a laser with a suitably high pulse energy and a pulse repetition rate matching the DMD refresh rate. Repetition rates for solid state lasers are increasing, and fibre lasers hold promise as high repetition rate sources in future. Here, we show that this system has sufficient acoustic sensitivity to be able to measure photoacoustic images. Movies hair $100 \%$ and hair_20\% show photoacoustic images of a knotted hair recovered using $100 \%$ and $20 \%$ patterns; movies ribbon_100\% and ribbon_10\% show photoacoustic images of a twisted ribbon phantom using $100 \%$ and $10 \%$ patterns.

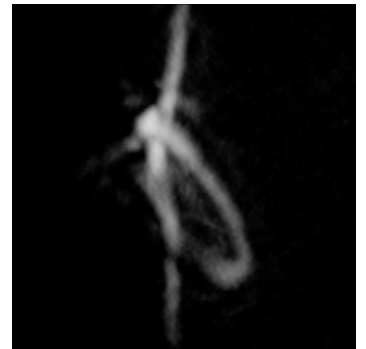

Movie 6: hair_100\%, http://

dx.doi.org/10.1117/12. 2081869.6

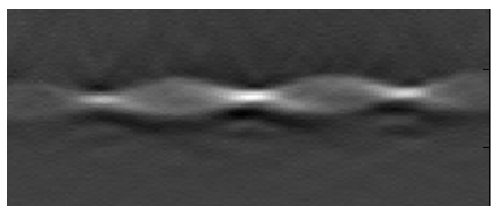

Movie 8: ribbon_100\%, http://

dx.doi.org/10.1117/12.2081869

.8

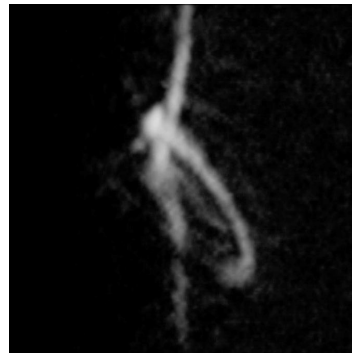

Movie 7: hair_20\%, http://

dx.doi.org/10.1117/12. 2081869.7

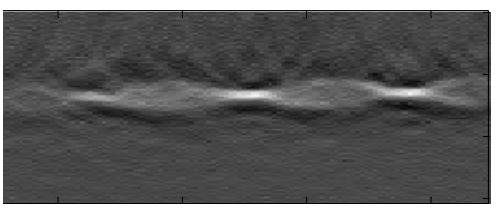

Movie 9: ribbon_10\%,

dx.doi.org/10.1117/12.208186

dx.doi.org/10.1117/12.208186 9.9
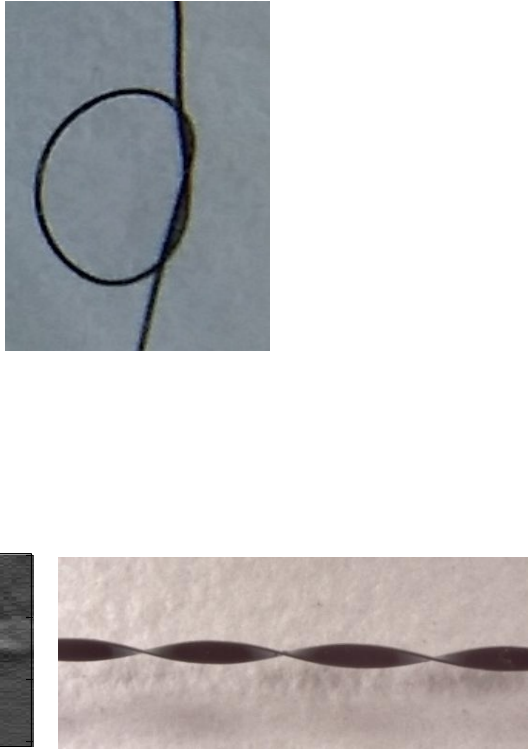


\section{ACKNOWLEDGMENTS}

The authors acknowledge support from the Engineering and Physical Sciences Research Council, UKand E uropean Union project FAMOS (FP7 ICT, Contract 317744)

\section{REFERENCES}

[1] Beard, P., "Biomedical Photoacoustic Imaging”, Interface Focus, 1, 602-31 (2011)

[2] Salim, M.S. et al., "Capacitive Micromachined Ultrasonic Transducers: Technology and Application", $J$. Medical Ultrasound, 20, 8-31 (2012)

[3] Wu, et al, "Very high frequency (beyond $100 \mathrm{MHz}$ ) PZT kerfless linear arrays", IEEE Trans. UFFC, 56, 23042310 (2009)

[4] Chen, J. et al., "Bandwidth improvement of LiNbO3 ultrasonic transducers by half-concaved inversion layer approach", Rev. Sci. Inst., 83, 114903 (2012)

[5] Li, H. et al., "A transparent broadband ultrasonic detector based on an optical micro-ring resonator for photoacoustic microscopy”, Scientific Reports, 4, 4496 (2014)

[6] Li, J., Taylor, A., Papakonstantinou, I., Zhang, E. and Beard, P. "Highly sensitive optical microresonator sensors for photoacoustic imaging", Proc. SPIE, 8943 (2014)

[7] Zhang, E. and Beard, P., "Broadband ultrasound field mapping system using a wavelength tuned, optically scanned focused laser beam to address a Fabry Perot polymer film sensor", IEEE Trans. UFFC, 53, 1330-8 (2006)

[8] Beard, P. and Mills, T., "Extrinsic optical fibre ultrasound sensor using a thin polymer film as a low finesse Fabry-Perot interferometer", Appl. Opt., 35, 663-675 (1996)

[9] Hamilton, J.D. et al., "High frequency optoacoustic arrays using etalon detection" IEEE Trans. UFFC, 47, 160$169(2000)$

[10] Hajireza, P., Krause,K., Brett, M. and Zemp, R., "Glancing angle deposited nanostructured film Fabry-Perot etalons for optical detection of ultrasound", Opt. Expr., 21, 6391-400 (2013)

[11] Laufer. J., Johnson, P., Zhang, E., Treeby, B., Cox, B.T., Pedley, B., and Beard, P. "In vivo preclinical photoacoustic imaging of tumor vasculature development and therapy", J. Biomed. Opt., 17, 056016 (2012)

[12] Shu, Y. et al., "One-dimensional Optoacoustic Receive Array Employing Parallel Detection and Video-rate Acquisition" Ultrasonics Symp. Proc., 2396-2399 (2010)

[13] Lamont, M. and Beard, P., "2D imaging of ultrasound fields using CCD array to map output of Fabry-Perot polymer film sensor", Elec. Lett., 42, 7-8 (2006)

[14] Cong, B. et al., "A fast acoustic field mapping approach based on Fabry-Perot sensor with high-speed camera", IEE J. Trans. Elec. Elec. Eng., 9, 477-483, (2014)

[15] Huynh, N., Zhang, E., Betcke, M., Arridge, S., Beard, P. and Cox, B.T., "Patterned interrogation scheme for compressed sensing photoacoustic imaging using a Fabry Perot planar sensor" Proc. SPIE, 8943, 894327 (2014)

[16] Texas Instruments, Using Lasers with DLP DMD Technology, (2008)

[17] Candès, E.J., Romberg, J.K., and Tao, T., "Stable signal recovery from incomplete and inaccurate measurements", Comm. Pure and Appl. Math., 59, 1207-1223 (2006)

[18] Do, T.T., Gan, L., Nguyen, N.H. and Tran, T.D. "Fast and efficient Compressive Sensing Using Structurally Random Matrices", IEEE Trans. Sig. Proc., 60, 139-154 (2012)

[19] Holger, R. "Compressive Sensing and Structured Random Matrices", Theoretical Foundations and Numerical Methods for Sparse Recovery. Berlin, Boston: DE GRUYTER, 1-92 (2010).

[20] Afonso, M. V., Bioucas-Dias, J. M., \& Figueiredo, M. A. "Fast image recovery using variable splitting and constrained optimization", IEEE Trans. Im. Proc., 19, 2345-2356 (2010). 